\title{
Mini invasive colorectal surgery: an update
}

\author{
Francesco Corcione $^{1} \cdot$ Nicolò de Manzini $^{2}$
}

Published online: 19 April 2016

(C) Italian Society of Surgery (SIC) 2016

Minimally invasive approach to colorectal disease nowadays involves most of the surgical communities in Italy and abroad. Many centers have started this surgery, but not everywhere has been standardized. Moreover, since the minimally invasive approach is innovative, it needs to be updated with the tips and tricks that are continuously added and ameliorated by expert laparoscopic surgeons.

This monographic volume tries therefore to reply to many open issues and to certify and codify what has been already acknowledged in clinical practice; we are witnesses of a dramatic change in the way of approaching this disease and we have the duty to offer the best available treatment to our patients. This volume may therefore represent a valid and detailed update for surgeons who are at the beginning of their experience as well as for those who want to improve their performance.

The latest medical technical advances, physiopathological knowledge, indications to surgical management of diverticular disease, description of standard as well as unusual surgical procedures; these are only some of the interesting topics of this volume. Coverage of all these aspects is accompanied by up-to-date references.

We would like to express many thanks to the eminent Italian and foreign Colleagues who enthusiastically joined this project and enriched our knowledge with their great experience. Thanks are also due to Springer for its valuable support and careful editing.

\section{Compliance with ethical standards}

Conflict of interest The authors declare that they have no conflict of interest.

Ethical standard All procedures in our paper were in accordance with the ethical standards of the institutional as well as national research committee and with the 1964 Helsinki declaration and its later amendments.

Research involving human participants and/or animals This articles does not contain any studies with human participants or animals performed by any of the authors.

Informed consent No informed consent is required.

Francesco Corcione

francesco.corcione@ospedalideicolli.it

1 Department of General Surgery, Monaldi Hospital A.O.R.N. dei Colli, Naples, Italy

2 Department of General Surgery, University Hospital of Trieste, Trieste, Italy 